COMMUNICATIONS IN

ANALYSIS AND GEOMETRY

Volume 13, Number 5, 939-961, 2005

\title{
Small knots and large handle additions
}

\author{
Ruifeng Qiu And Shicheng Wang ${ }^{1}$
}

\begin{abstract}
We construct a hyperbolic 3-manifold $M$ (with $\partial M$ totally geodesic) which contains no essential closed surfaces, but for any even integer $g>0$, there are infinitely many separating slopes $r$ on $\partial M$ so that $M[r]$, the 3-manifold obtained by attaching 2-handle to $M$ along $r$, contains an essential separating closed surface of genus $g$ and is still hyperbolic. The result contrasts sharply with those known finiteness results for the cases $g=0,1$. Our 3-manifold $M$ is the complement of a simple small knot in a handlebody.
\end{abstract}

\section{Introduction.}

All manifolds in this paper are orientable. All submanifolds are embedded and proper $(F \subset M$ is proper if $F \cap \partial M=\partial F)$, unless otherwise specified. A connected 1-manifold (an arc or a circle) on a surface $F$ is non-trivial if it does not separate a disc from $F$.

Let $M$ be a compact 3 -manifold with the boundary $\partial M \neq \emptyset, F$ be a surface in $M$ which is not the 2-sphere $S^{2}$. Say $F$ is incompressible if a circle $c \subset F$ bounds a disk in $M$ implies that $c$ bounds a disc in $F$. Say a surface in $M$ is essential if either it is incompressible and is not parallel to a sub-surface of $\partial M$, or it is a 2-sphere which does not bound a 3-ball in $M$. Say a 3-manifold $M$ is irreducible if each 2-sphere in $M$ bounds a 3-ball. Say $M$ is $\partial$-irreducible if $\partial M$ is incompressible. Say $M$ is atoroidal if it contains no essential tori; Say $M$ is anannular if it contains no essential annuli.

Say a 3-manifold $M$ is simple if $M$ is irreducible, $\partial$-irreducible, anannular and atoroidal. Suppose $M$ is a simple 3-manifold with $\partial M \neq \emptyset$. By Thurston's theorem, $M$ admits a complete finite volume hyperbolic structure with totally geodesic boundary (with torus components in $\partial M$ removed) [8]. A knot $K$ in $M$ is simple if $M_{K}$, the complement of $K$ in $M$, is simple. A 3-manifold $M$ is small if $M$ contains no essential closed surface. A knot $K$ in $M$ is small if $M_{K}$ is small.

\footnotetext{
${ }^{1}$ Both authors are supported by NSFC, the second author is also supported by MOSTC.
} 
A (separating) slope $r$ in $\partial M$ is the isotopy class of a non-oriented nontrivial (separating) circle in $\partial M$. We denote by $M[r]$ the manifold obtained by adding a 2-handle to $M$ along a regular neighborhood of $r$ in $\partial M$ and then capping off spherical components with 3-balls. Specially, if $r$ lies in a torus component of $\partial M$, this operation is known as Dehn filling.

Essential surface is a basic tool to study 3-manifolds and handle addition is a basic method to construct 3-manifolds. A central topic connecting those two aspects in 3-manifold topology is the following:

Question 1.1. Let $M$ be a simple 3-manifold with $\partial M \neq \emptyset$ which contains no essential closed surface of genus $g$. How many slopes $r \subset \partial M$ are there so that $M[r]$ contains an essential closed surface of genus $g$ ?

Remark on Question 1.1. The mapping class group of a simple 3manifold $M$ with $\partial M \neq \emptyset$ is finite. The question is asked only for simple 3-manifolds to avoid possibly infinitely many slopes produced from Dehn twists along essential discs or annuli. The main result in this paper is the following:

Theorem 1.2. There is a simple small knot $K$ in the handlebody $H$ of genus 3 such that for any even integer $g>0$, there are infinitely many separating slopes $r$ in $\partial H$ so that $H_{K}[r]$ contains an essential separating closed surface of genus $g$. Moreover, those $H_{K}[r]$ are still simple.

\section{Remarks on Theorem 1.2}

(1) Suppose $M$ is a simple 3 -manifolds with $\partial M \neq \emptyset$.

(i) $\partial M$ is a torus. Thurston's pioneering result claims that there are at most finitely many slopes $r$ on $\partial M$ so that $M[r]$ is not hyperbolic [8], hence the number of slopes are finite in Question 1.1 when $g=0,1$. The sharp upper bound of such slopes are given by Gordon and Luecke and by Gordon when $g=0,1$, see [2] for a survey. Hatcher proved the number of slopes in Question 1.1 is finitely many for all $g$ [3].

(ii) $\partial M$ has genus $>1$. Scharlemann and Y-Q Wu [7] have shown that if $g=0,1$, then there are only finitely many separating slopes $r$ so that $M[r]$ contains an essential closed surface of genus $g$. Very recently, Lackenby [5] generalized Thurston's finiteness result to handlebody attaching, that is to adding 2-handles simultaneously. He proved that for a hyperbolic 3 -manifold $M$, there is a finite set $\mathcal{C}$ of exceptional circles on $\partial M$ so that attaching a handlebody to $M$ is still hyperbolic if none of those circles is attached to a meridian disc of the handlebody. 
Theorem 1.2 and those finiteness results of [8], [3], [7] and [5] give a global view about the answer of Question 1.1. In particular, those finiteness results of [8], [3] and [7] do not hold in general. We think the example in Theorem 1.2 also indicates that the finiteness result of [5] does not hold in general (a working project of the authors).

(2) It is unusual to the authors that a given manifold $M$ provides nonfiniteness answer to Question 1.1 for all even genus $g \geq 2$. From an aesthetic point of view, one may wonder if there is a manifold that provides nonfiniteness answer to Question 1.1 for all genus $g \geq 2$. We think that the answer is positive. In this case, the knot $K$ is complicated and then the proof of that $H_{K}$ is small will be much more difficult (a working project of the authors).

(3) Without handle addition, the 3-manifold $M$ itself in Theorem 1.2 is interesting independently. First, the construction of the small knot in Theorem 1.2 can be modified to provided infinitely many small knots in handlebodies of any genus $g>1$ (a working project of the authors). To our knowledge, no examples of simple small knots in the handlebody of genus $>1$ were explicitly presented before. Secondly, $M$ provides a hyperbolic 3manifold with totally geodesic boundary which splits over essential surfaces of genus $g$ in infinitely many different ways for each even $g>0$.

Remarks on the Proof of Theorem 1.2 and the organization of the paper. In Section 2, we construct a knot $K$ and infinitely many separating surfaces $S_{g, l}$ of genus $g$ for each even $g>0$ in the handlebody $H$ of genus 3, such that all those surfaces $S_{g, l}$ are disjoint from $K$ and have connected boundaries. Those $\partial S_{g, l}$ will serve as the slopes $r$ in Theorem 1.2. Some elementary properties of $S_{g, l}$ and of $K$ are also described in Section 2. Let $\hat{S}_{g, l} \subset H_{K}\left[\partial S_{g, l}\right]$ be the closed surface obtained by capping off $\partial S_{g, l}$ with a disk. In Section 3, we will prove that $\hat{S}_{g, l}$ is incompressible in $H_{K}\left[\partial S_{g, l}\right]$ as well as that $\partial S_{g, l}$ and $\partial S_{g, l^{\prime}}$ are not isotopic in $\partial H$ when $l \neq l^{\prime}$. Sections 4 and 5 are devoted to proving that the knot $K$ is simple and small.

A result in [4] is quoted in Section 3, which is a crucial step for the proof of Proposition 3.2, and a result in [1] is quoted in Section 4, which is used to shorten the argument of Case 2 in the proof of Lemma 4.4. Up to those two results and the knowledge in the beginning of standard textbooks of elementary algebraic topology, combinatorial groups and 3-manifolds, the paper is self-contained. Even so, the argument of Case 1 (2) in the proof of Lemma 4.4 is initiated by Gordon-Litherland in the mid 1980's. 


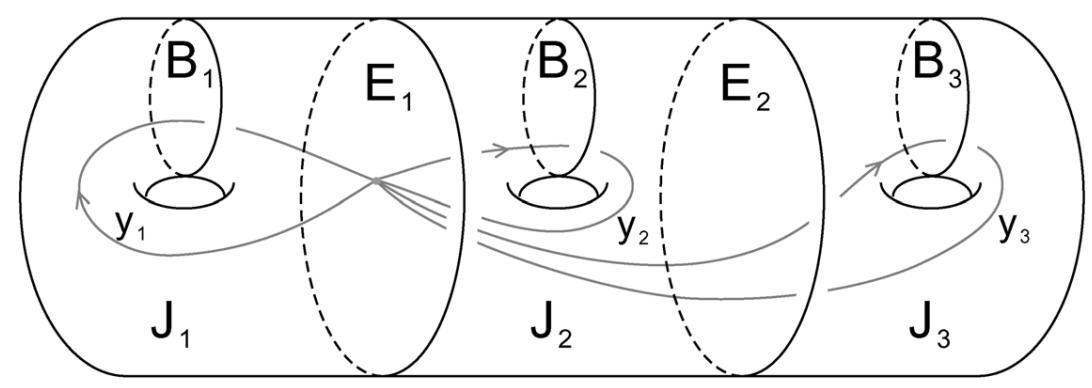

Figure 1.

\section{Construction of the surfaces $S_{g, l}$ and the knot $K$ in $H$.}

Suppose $X_{1}$ and $X_{2}$ are connected proper sub-manifolds of $M$ with complementary dimensions and meeting tranversely. Let $\left\|X_{1}, X_{2}\right\|$ be the absolute value of their algebraic intersection number. Since all manifolds are orientable, $\left\|X_{1}, X_{2}\right\|$ is well defined. For a compact manifold $X,|X|$ denotes the number of components of $X$. If $X$ is an arc or an annulus, we often use $\partial_{1} X$ to denote one component of $\partial X$ and $\partial_{2} X$ to denote another.

Let $H$ be the handlebody of genus 3 . Let $\left\{B_{1}, B_{2}, B_{3}\right\}$ be a set of basis disks of $H$, and $\left\{E_{1}, E_{2}\right\}$ be two separating disks of $H$ which separate $H$ into three solid tori $J_{1}, J_{2}$ and $J_{3}$. See Figure 1.

The orientable surface $S_{g}$ of even genus $g>0$ with $\left|\partial S_{g}\right|=1$ can be presented as in Figure 2 (where $g=4$ ). Each surface $S_{g, l}$ we are going to construct in $H$ can be viewed as a properly embedded image of $S_{g}$, where the disk in Figure 2 is sent to $E_{1}$ (approximately) and the 1-handle ended at $v_{i}$ and $u_{i}$ is sent to the 1-handle $N\left(\alpha_{i}\right)$ attached to $E_{1}$, which will be shown in Figures 3 and 4

Remark on Figure 2 In Figure 2, if we attach $g$ 1-handles on each side of the disc for odd $g$ in the same way, we get a surface of genus $g-1$ with three boundary components rather than a surface of genus $g$ with one boundary component.

Let $C$ be a closed curve in $\partial H$ (with one self-intersection) as in Figure 3. Then $\partial E_{1} \cup \partial E_{2}$ separates $C$ into eight embedded arcs $c_{1}, \ldots, c_{8}$, where $c_{3}, c_{7} \subset J_{1}$ with $\left\|\partial B_{1}, c_{7}\right\|=3,\left\|\partial B_{1}, c_{3}\right\|=1 ; c_{2}, c_{4}, c_{6}, c_{8} \subset J_{2}$ with $\left\|\partial B_{2}, c_{4}\right\|=1,\left\|\partial B_{2}, c_{6}\right\|=3,\left\|\partial B_{2}, c_{2}\right\|=\left\|\partial B_{2}, c_{8}\right\|=0 ; c_{1}, c_{5} \subset J_{3}$ with $\left\|\partial B_{3}, c_{1}\right\|=3,\left\|\partial B_{3}, c_{5}\right\|=1$.

Let $u_{1}, \ldots, u_{2 g}, v_{1}, \ldots, v_{2 g}$ be $4 g$ points located on $\partial E_{1}$ in the cyclic order 


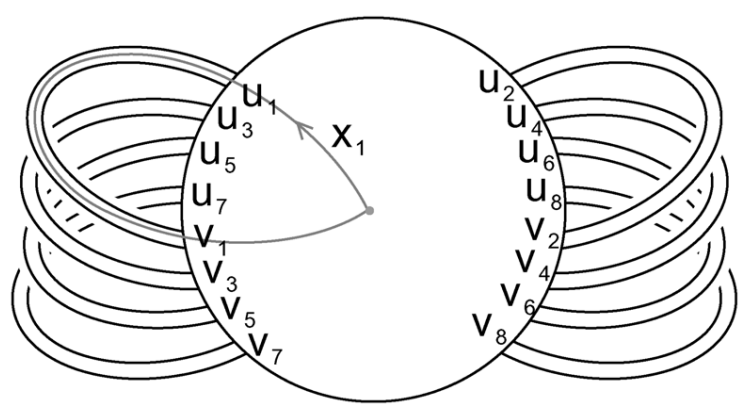

Figure 2 .

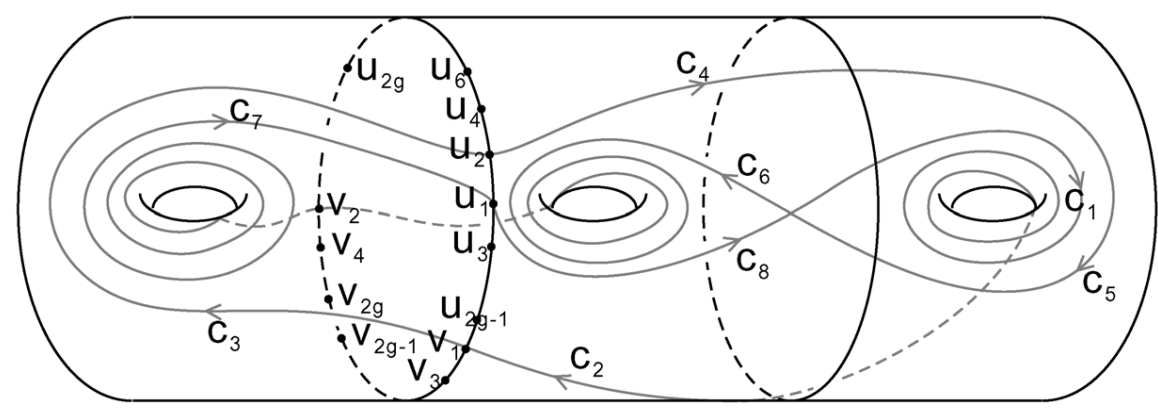

Figure 3.

$u_{1}, u_{3}, \ldots, u_{2 g-3}, u_{2 g-1}, v_{1}, v_{3}, \ldots, v_{2 g-3}, v_{2 g-1}, v_{2 g}, v_{2 g-2}, \ldots, v_{4}, v_{2}, u_{2 g}$, $u_{2 g-2}, \ldots, u_{4}, u_{2}$ as in Figure 3 (see also Figure 2).

By the order of those points, we can assume that the isotopy has been made so that $\partial\left(c_{8} \cup c_{1} \cup c_{2}\right)=\left\{u_{1}, v_{1}\right\}, \partial c_{3}=\left\{v_{1}, u_{2}\right\}, \partial c_{7}=\left\{v_{2}, u_{1}\right\}$. Then pick a proper $\operatorname{arc} c_{*}$, (resp. $\left.c_{\#}\right)$ in $\partial H \cap\left(J_{2} \cup J_{3}\right)$ connecting $v_{3}$ and $v_{2}$ (resp. $u_{2}$ and $\left.u_{3}\right)$ as in Figure 4 , where $\left\|c_{*}, \partial B_{2}\right\|=l, l \geq 3$. By construction, intc $_{\#} \cap C=\emptyset$ and $c_{*} \cap C \neq \emptyset$.

Now, we define oriented arcs on $\partial H$ to connect some pairs in $\left\{u_{i}, v_{j} ; i, j=1, \ldots, 2 g\right\}$ as follows: First let $\overline{u_{1} v_{1}}=c_{8} \cup c_{1} \cup c_{2}, \overline{v_{1} u_{2}}=c_{3}$, $\overline{v_{2} u_{1}}=c_{7}, \overline{u_{2} u_{3}}=c_{\#}, \overline{v_{3} v_{2}}=c_{*}$. Then, let $\overline{v_{2 i} u_{2 i-1}}$ and $\overline{v_{2 i-1} u_{2 i}}$ be proper arcs on $\partial H \cap J_{1}$ parallel to $c_{7}$ and $c_{3}$ respectively, $i=2, \ldots, g$ and $\overline{u_{2 i} u_{2 i+1}}$ and $\overline{v_{2 i+1} v_{2 i}}$ be a proper arcs on $\partial H \cap\left(J_{2} \cup J_{3}\right)$ parallel to $c_{\#}$ and $c_{*}$ respectively, $i=2, \ldots, g-1$. See $\overline{u_{3} v_{4}}$ and $\overline{u_{4} u_{5}}$ in Figure 4. Now define 


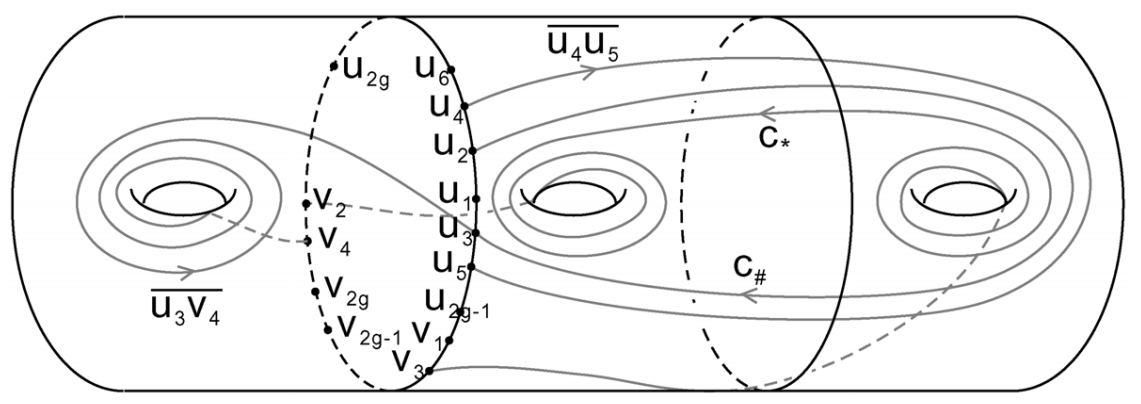

Figure 4.

$$
\alpha_{1}=\overline{u_{1} v_{1}}
$$

and for $1<4 k+j \leq 2 g, j=1,2,3,4$,

$$
\begin{aligned}
& \alpha_{4 k+1}=\overline{u_{4 k+1} u_{4 k}} \cup \alpha_{4 k} \cup \overline{v_{4 k} v_{4 k+1}}, \\
& \alpha_{4 k+2}=\overline{v_{4 k+2} u_{4 k+1}} \cup \alpha_{4 k+1} \cup \overline{v_{4 k+1} u_{4 k+2}} \text {, } \\
& \alpha_{4 k+3}=\overline{v_{4 k+3} v_{4 k+2}} \cup \alpha_{4 k+2} \cup \overline{u_{4 k+2} u_{4 k+3}} \text {, } \\
& \alpha_{4 k+4}=\overline{u_{4 k+4} v_{4 k+3}} \cup \alpha_{4 k+3} \cup \overline{u_{4 k+3} v_{4 k+4}} \text {. }
\end{aligned}
$$

Hence, $\alpha_{k-1} \subset \alpha_{k}$ is an increasing sequence of embedded arcs on $\partial H$.

Let $\alpha \subset \partial H$ be an arc which meets $\partial S$ exactly in its two ends for a proper separating surfaces $S \subset H$. The resulting proper surface by tubing $S$ along $\alpha$ in $H$, denoted by $S(\alpha)$, is obtained by first attaching 2-dimensional 1handle $N(\alpha) \subset \partial H$ to $S$, then making the surface $S \cup N(\alpha)$ to be proper, that is, pushing the interior of $S \cup N(\alpha)$ into the interior of $H$. The image of $N(\alpha)$ after the pushing is still denoted by $N(\alpha)$. Since $S$ is orientable and separating, it is a direct observation that $S(\alpha)$ is still orientable and separating.

Since $\alpha_{1}$ meets $E_{1}$ exactly in its two ends, we do tubing of $E_{1}$ along $\alpha_{1}$ to get $E_{1}\left(\alpha_{1}\right)$. Now, $\alpha_{2}$ meets $E_{1}\left(\alpha_{1}\right)$ exactly in its two ends, we do tubing of $E_{1}\left(\alpha_{1}\right)$ along $\alpha_{2}$ to get $E_{1}\left(\alpha_{1}, \alpha_{2}\right)=E_{1}\left(\alpha_{1}\right)\left(\alpha_{2}\right)$, where the tube $N\left(\alpha_{2}\right)$ is thinner and closer to $\partial H$ so that it goes over the tube $N\left(\alpha_{1}\right)$. Hence, $E_{1}\left(\alpha_{1}, \alpha_{2}\right)$ is a proper embedded surface. Repeating this process by tubing along $\alpha_{3}, \ldots, \alpha_{2 g}$ in order, we get a surface $E_{1}\left(\alpha_{1}, \ldots, \alpha_{2 g}\right)$, denoted by $S_{g, l}$, in $H$. Clearly, $S_{g, l}$ is a proper embedding of $S_{g}$ into $H$ for each even $g>0$. We survey this fact as 


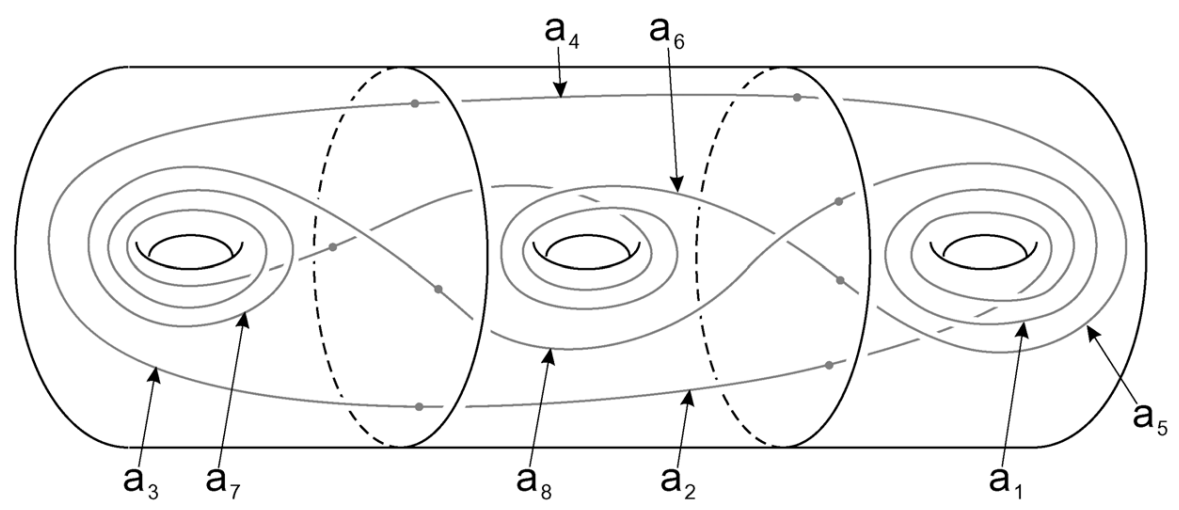

Figure 5 .

Lemma 2.1. $S_{g, l}$ is an orientable separating surface in $H$. Moreover, $S_{g, l}$ is of genus $g$ with $\left|\partial S_{g, l}\right|=1$ for even $g>0$ (and of genus $g-1$ with $\left|\partial S_{g, l}\right|=3$ for odd $\left.g\right)$.

In the construction of $S_{g, l}$ for all $g, l$, we may assume that (i) the positions of the $\operatorname{arcs} \alpha_{1}, \alpha_{2}$ are fixed; (ii) each tube $N\left(\alpha_{i}\right)$ has distance $\delta / i$ from $\alpha_{i}$ for some $\delta>0$. By (i) and (ii), we have (iii) $N\left(\alpha_{1}\right), N\left(\alpha_{2}\right)$ and the part of $N\left(\alpha_{3}\right)$ that goes over $N\left(\alpha_{2}\right)$ are fixed for all $g, l$.

Now, our knot $K$ is obtained by pushing $C$ into the interior of $H$ along the inward normal direction of $\partial H$ in the following way: (iv) first push the arc $c_{7} \cup c_{8} \cup c_{1} \cup c_{2} \cup c_{3}$ to stay between $N\left(\alpha_{2}\right)$ and $N\left(\alpha_{3}\right)$, (v) then push the arc $c_{4} \cup c_{5} \cup c_{6}$ so that it has distance larger than $\delta / 3$ from $\partial H$ and is disjoint from $N\left(\alpha_{1}\right)$. Below, we use $a_{i}$ to denote the image of $c_{i}$ after pushing. Then $E_{1} \cup E_{2}$ separates $K$ into $8 \operatorname{arcs} a_{1}, \ldots, a_{8}$. See Figure 5 for $K, a_{i} \subset H$, where $a_{6}$ is crossing under $a_{8}$, and $\left\|a_{i}, B_{k}\right\|($ in $H)=\left\|c_{i}, \partial B_{k}\right\|($ in $\partial H), i=1, \ldots, 8$ and $k=1,2,3$.

Lemma 2.2. $K \cap S_{g, l}=\emptyset$ for all $g, l$.

Proof. By (iii) and (iv), the part $a_{7} \cup a_{8} \cup a_{1} \cup a_{2} \cup a_{3}$ of $K$ is disjoint from $S_{g, l}$. By (ii), (iii) and (v), the part $a_{4} \cup a_{5} \cup a_{6} \subset J_{2} \cup J_{3}$ of $K$ is also disjoint from $S_{g, l}$. Hence $K \cap S_{g, l}=\emptyset$ for all $g, l$.

Let $N(K)=K \times D$ be the regular neighborhood of $K$ in $H$ such that (i) $S_{g, l} \subset H_{K}=H-\operatorname{int} N(K)$ for all $g, l$, (ii) the product structure has been 


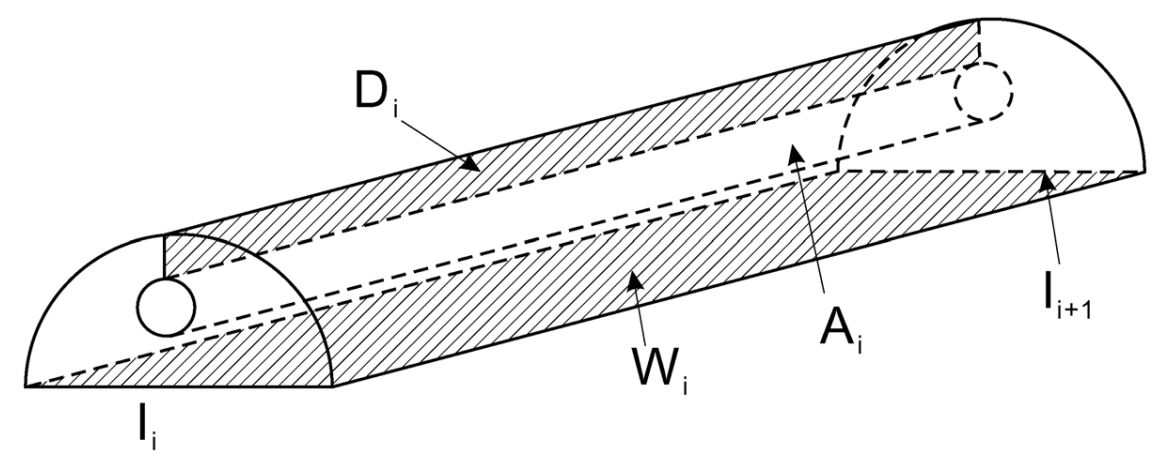

Figure 6.

adjusted so that $\cup_{i=1}^{8} \partial a_{i} \times D \subset E_{1} \cup E_{2}$. Let $F_{j}=E_{j}-\operatorname{int} N(K), j=1,2$; $M_{k}=H_{K} \cap J_{k}, k=1,2,3$; and $T=\partial(K \times D)$. Then $F_{1} \cup F_{2}$ separates $T$ into eight annuli $A_{1}, \ldots, A_{8}$, where $A_{i}=a_{i} \times \partial D$. Moreover, $K$ and $C$ bound a non-embedded annulus in $H$ (the trace of pushing $C$ to $K$ ) which is cut by $E_{1} \cup E_{2}$ into eight disk $D_{i *}$, with $a_{i} \subset \partial D_{i *}, i=1, \ldots, 8$. Suppose $a_{i} \subset M_{k}$, then $D_{i}=D_{i *} \cap M_{k}$ is a proper disc in $M_{k}$. Let $W_{i}=\overline{\partial N\left(D_{i} \cup A_{i}\right)-\partial M_{k}}$, where $N\left(D_{i} \cup A_{i}\right)$ is a regular neighborhood of $D_{i} \cup A_{i}$ in $M_{k}$. Then $W_{i}$ is a proper separating disk in $M_{k}$. Each $W_{i}$ intersects $F_{1} \cup F_{2}$ in two arcs $l_{i}$ and $l_{i+1}$. Note $W=\cup_{i \neq 6} W_{i}$ is still a (non-proper) disc. We use $\mu$ to denote the meridian slope on $T$. See Figure 6 for $A_{i}, D_{i}, W_{i}, l_{i}, l_{i+1} \subset M_{k}$.

The following facts about $K$ and $a_{i}$, which are based on Figure 5 and whose proofs involve only elementary algebraic topology rather than 3manifold topology, will be used in Sections 4 and 5 .

Lemma 2.3. (1) $K$ is not contractible in $H$.

(2) Suppose $a_{i}, a_{j} \subset J_{k}$. There is no relative homotopy on $\left(J_{k}, E_{1} \cup E_{2}\right)$ which either sends $a_{i}$ to $E_{1} \cup E_{2}$; or sends $a_{i}$ to $a_{j}$ unless $(i, j)$ is $(2,8)$.

(3) Suppose $a_{i}, a_{j} \subset J_{k}$. The meridians of $A_{i}$ and $A_{j}$ are not homotopic in $M_{k}$.

(4) Suppose $B$ is a proper disc of $J_{k}$ with $\left|B \cap E_{j}\right| \leq 1, j=1,2$. If $\left|B \cap\left(\cup_{a_{i} \subset J_{k}} a_{i}\right)\right|<3-\left|B \cap\left(E_{1} \cup E_{2}\right)\right|$, then $B$ separates a 3-ball from $J_{k}$.

(5) There is no annulus $A \subset H$ such that ( $i) \partial_{1} A=K$ and $\partial_{2} A \subset \partial H$, (ii) each component of $A \cap\left(E_{1} \cup E_{2}\right)$ is non-trivial in $A$.

Proof. The proofs of (1), (2), and (3) are direct. 
(4) If $B$ is a separating disk in $J_{k}$, then $B$ separates a 3 -ball from $J_{k}$, since $J_{k}$ is a solid torus. So we need only to show that each non-separating disk in $J_{k}$ does not meet the inequality in (4).

Note $B \cap E_{j}$ is either an arc or the empty-set. We suppose $B$ is a non-separating disk in $J_{2}$ which meets each $E_{j}$ in an arc $d_{j}, j=1,2$ (the remaining cases are more direct). Let $b_{j}$ be an arc in $E_{j}$ connecting the two endpoints of $a_{6}$ and $a_{8}$. Then $c=b_{1} \cup a_{6} \cup b_{2} \cup a_{8}$ is a circle which goes around $J_{3}$ three times. Hence

$$
\begin{aligned}
3=\|B, c\| & \leq\left\|B, a_{6} \cup a_{8}\right\|+\left\|\partial B, b_{1}\right\|+\left\|\partial B, b_{2}\right\| \\
& =\left\|B, a_{6} \cup a_{8}\right\|+\left\|d_{1}, b_{1}\right\|+\left\|d_{2}, b_{2}\right\| .
\end{aligned}
$$

By the Jordan Curve Theorem, ||$d_{j}, b_{j} \| \leq 1=\left|B \cap E_{j}\right|$. Hence,

$$
\left|B \cap\left(\cup_{a_{i} \subset J_{k}} a_{i}\right)\right| \geq|| B, b_{1} \cup a_{6} \cup b_{2} \cup a_{8}|| \geq 3-\left|B \cap\left(E_{1} \cup E_{2}\right)\right| .
$$

(5) Otherwise, there is an annulus $A$ that meets (i) and (ii) in (5). Then by (1), $A$ is cut by $E_{1} \cup E_{2}$ into eight rectangles $R_{i}, i=1, \ldots, 8$, each $R_{i}$ has two opposite sides in $E_{1} \cup E_{2}$ and remaining two sides $a_{i}$ in $K$ and $a_{i}^{*} \subset \partial H$. Let $b_{i}$ be an arc in $E_{1} \cup E_{2}$ connecting $\partial a_{i}^{*}$, and denote the circle $b_{i} \cup a_{i}^{*} \subset J_{k}$ by $e_{i}, i=1,3,7,5$. In a basis of $H_{1}\left(\partial J_{3}, Z\right), e_{1}$ and $e_{5}$ have coordinates $(3, p)$ and $(1, q)$ respectively, and hence $\left\|b_{1}, b_{5}\right\|=\| e_{1}, e_{5}||=|3 q-p| \neq 0$, since $p$ and 3 are co-prime. It follows that $\partial b_{1}=\partial a_{1}^{*}$ and $\partial b_{5}=\partial a_{5}^{*}$ are alternating on $\partial E_{2}$. By the same reason, $\partial a_{3}^{*}$ and $\partial a_{7}^{*}$ are alternating on $\partial E_{1}$.

Now, back to $J_{2}, \partial a_{i}^{*}$ 's have the cyclic order $4,8,2,6$ in $\partial E_{1}$, and the cyclic order $4,8,6,2$ in $\partial E_{2}$. Hence, there are four disjoint arcs on $E_{1} \cup E_{2}$ such that the two with $a_{4}^{*} \cup a_{8}^{*}$ form a circle $e_{4,8}$ on $\partial J_{2}$, and the other two with $a_{2}^{*} \cup a_{6}^{*}$ form a circle $e_{2,6}$ on $\partial J_{2}$, moreover $e_{2,6}$ and $e_{4,8}$ are disjoint, therefore they are parallel on $\partial J_{2}$. But in a basis of $H_{1}\left(\partial J_{2}, Z\right)$, those two circles have coordinates $(3, p)$ and $(1, q)$, and $\left\|e_{2,6}, e_{4,8}\right\|=|3 q-p| \neq 0$, since 3 and $p$ are coprime. A contradiction.

\section{Proof of Theorem 1.2 by assuming that $K$ is simple and small.}

In this section, $g>0$ will be even integer. By Lemma 2.1, let $\hat{S}_{g, l} \subset$ $H_{K}\left[\partial S_{g, l}\right] \subset H\left(\partial S_{g, l}\right)$ the surface obtained by capping off the boundary of $S_{g, l}$ with a disk. Then $\hat{S}_{g, l}$ is a closed surface of genus $g$.

Now, Theorem 1.2 follows from the following two propositions (the "Moreover" part of Theorem 1.2 follows directly from [7]). 
Proposition 3.1. $K \subset H$ is a simple and small knot.

Proposition 3.2. (1) $\hat{S}_{g, l}$ is incompressible in $H_{K}\left[\partial S_{g, l}\right]$. $l \neq l^{\prime}$.

(2) for given $g, \partial S_{g, l}$ and $\partial S_{g, l^{\prime}}$ are not the same slope in $\partial H_{K}$ when

We choose the center of $E_{1}$ as the common base point for the fundamental groups of $H$ and of all surfaces $S_{g, l}$. Now, $\pi_{1}(H)$ is the free group of rank three generated by $y_{1}, y_{2}, y_{3}$ indicated in Figure 1 . and $\pi_{1}\left(S_{g, l}\right)$ is the free group of rank $2 g$ generated by $x_{1}, \ldots, x_{2 g}$, where $x_{i}$ is the generator given by $\alpha_{i}$ and two arcs in $E_{1}$ (see Figure 2). Let $\phi: S_{g, l} \rightarrow H$ be the inclusion (precisely $\phi$ should be $\phi_{g, l}$, we omit sub-index without making confusion), $\phi_{*}: \pi_{1}\left(S_{g, l}\right) \rightarrow \pi_{1}(H)$ be the induced homomorphism. It is easy to see from Figures 1, 3 and 4,

$$
\tilde{c}_{3}=y_{1}, \tilde{c}_{7}=y_{1}^{3}, \tilde{c}_{\#}=y_{2} y_{3}, \tilde{c}_{*}=y_{3}^{-3} y_{2}^{-l}
$$

where $\tilde{c}_{3} \in \pi_{1}(H)$ is given by $c_{3}$ and two arcs in $E_{1}$ and so on.

Recall that $\overline{v_{2 i} u_{2 i-1}}, \overline{v_{2 i-1} u_{2 i}} \overline{u_{2 i} u_{2 i+1}}$ and $\overline{v_{2 i+1} v_{2 i}}$ are parallel copies of $c_{7}, c_{3}, c_{\#}$ and $c_{*}$ respectively. One can read $\phi_{*}\left(x_{i}\right)$ directly as words in $y_{1}, y_{2}, y_{3}$ by $(2.0)-(2.4)$ and $(*)$. They are:

$$
\phi_{*}\left(x_{1}\right)=y_{3}^{3},
$$

and for $1<4 i+j \leq 2 g, j=1,2,3,4$,

$$
\begin{aligned}
& \phi_{*}\left(x_{4 i+1}\right)=y_{3}^{-1} y_{2}^{-1} \phi_{*}\left(x_{4 i}\right) y_{2}^{l} y_{3}^{3}=\left(w_{1}^{-1} w_{2}\right)^{i} y_{3}^{3}\left(w_{1} w_{2}^{-1}\right)^{i} \\
& \phi_{*}\left(x_{4 i+2}\right)=y_{1}^{3} \phi_{*}\left(x_{4 i+1}\right) y_{1}=y_{1}^{3}\left(w_{1}^{-1} w_{2}\right)^{i} y_{3}^{3}\left(w_{1} w_{2}^{-1}\right)^{i} y_{1}, \\
& \phi_{*}\left(x_{4 i+3}\right)=y_{3}^{-3} y_{2}^{-l} \phi_{*}\left(x_{4 i+2}\right) y_{2} y_{3}=w_{2}\left(w_{1}^{-1} w_{2}\right)^{i} y_{3}^{3}\left(w_{1} w_{2}^{-1}\right)^{i} w_{1}, \\
& \phi_{*}\left(x_{4 i+4}\right)=y_{1}^{-1} \phi_{*}\left(x_{4 i+3}\right) y_{1}^{-3}=y_{1}^{-1} w_{2}\left(w_{1}^{-1} w_{2}\right)^{i} y_{3}^{3}\left(w_{1} w_{2}^{-1}\right)^{i} w_{1} y_{1}^{-3} \\
& \text { where } w_{1}=y_{1} y_{2} y_{3} \text { and } w_{2}=y_{3}^{-3} y_{2}^{-l} y_{1}^{3} .
\end{aligned}
$$

Obviously

$$
\begin{aligned}
& \left(w_{1} w_{2}^{-1}\right)^{j} w_{1}\left(w_{1}^{-1} w_{2}\right)^{i}=w_{2}\left(w_{1}^{-1} w_{2}\right)^{i-j-1} \text { if } i>j \text { and } \\
& \left(w_{1} w_{2}^{-1}\right)^{j} w_{1}\left(w_{1}^{-1} w_{2}\right)^{i}=\left(w_{1} w_{2}^{-1}\right)^{j-i} w_{1} \text { if } i \leq j . \\
& \left(w_{1} w_{2}^{-1}\right)^{j} w_{2}\left(w_{1}^{-1} w_{2}\right)^{i}=w_{2}\left(w_{1}^{-1} w_{2}\right)^{i-j} \text { if } i \geq j \text { and } \\
& \left(w_{1} w_{2}^{-1}\right)^{j} w_{2}\left(w_{1}^{-1} w_{2}\right)^{i}=\left(w_{1} w_{2}^{-1}\right)^{j-i-1} w_{1} \text { if } i<j .
\end{aligned}
$$

Lemma 3.3. (1) $S_{g, l}$ is incompressible in $H$.

(2) for given $g, \partial S_{g, l}$ and $\partial S_{g, l^{\prime}}$ are not in the same slope in $\partial H$ if $l \neq l^{\prime}$. 
Proof. By (3.5), the right sides of (3.0)-(3.4) are reduced words in < $y_{1}, y_{2}, y_{3}>$. Now, we present $\pi_{1}\left(S_{l, g}\right)$ as the free product $G_{1} * G_{2}$, where $G_{1}=<x_{1}, x_{3}, \ldots, x_{2 g-1}>$ and $G_{2}=<x_{2}, x_{4}, \ldots, x_{2 g}>$.

(1) We need only to show that $\phi_{*}: \pi_{1}\left(S_{g, l}\right) \rightarrow \pi_{1}(H)$ is injective.

For each $w_{2} \in G_{2}$, we may suppose that $w_{2}$ is a reduced form in < $x_{2}, \ldots, x_{2 g}>$. Now, we can present $\phi_{*}\left(w_{2}\right)$ as a word in $\left\langle y_{1}, y_{2}, y_{3}\right\rangle$ by first replacing each $x_{2 i}^{ \pm l} \in w_{2}$ by $\phi_{*}\left(x_{2 i}\right)^{ \pm l}$ and then applying (3.2) and (3.4). By (3.5), (3.6), (3.7) and obvious cancellations, one can get a reduced form of $\phi_{*}\left(w_{2}\right)$ in $\left\langle y_{1}, y_{2}, y_{3}\right\rangle$. Indeed by an induction on the length of the reduced form $w_{2}$, it is easy to see that if $w_{2} \neq 1$, then $\phi_{*}\left(w_{2}\right) \neq 1$ and $\phi_{*}\left(w_{2}\right)$ has the reduced form started from and ended by the non-zero powers of $y_{1}$. Similarly, one can argue that for $1 \neq w_{1} \in G_{1}, \phi_{*}\left(w_{1}\right) \neq 1$ and $\phi_{*}\left(w_{1}\right)$ has the reduced form started from and ended by the non-zero powers of $y_{3}$ and $y_{2}$.

Now, present each $1 \neq w \in G_{1} * G_{2}$ in a reduced form $g_{1}, g_{2}, \ldots, g_{n}$ of $G_{1} * G_{2}$, and each $g_{i}$ in a reduced form in $G_{1}$ or $G_{2}$. It is clear that $\phi_{*}(w) \neq 1$.

(2) For given $g, l$, the conjugacy class corresponding to $\partial S_{g, l}$ in $\pi_{1}\left(S_{g, l}\right)$ can be presented by a reduced word below (see Figure 2): $(* *)$

$x_{1} x_{3}^{-1} \ldots x_{2 g-3} x_{2 g-1}^{-1} x_{1}^{-1} x_{3} \ldots x_{2 g-3}^{-1} x_{2 g-1} x_{2 g}^{-1} x_{2 g-2} \ldots x_{4}^{-1} x_{2} x_{2 g} x_{2 g-2}^{-1} \ldots x_{4} x_{2}^{-1}$

Now, we can present $\phi_{*}\left(\left[\partial S_{g, l}\right]\right)$ in $\pi_{1}(H)$ as a word of $<y_{1}, y_{2}, y_{3}>$ by $(* *)$ and (3.0)-(3.4). Then doing cancellations to get the reduced form of $\phi_{*}\left(\left[\partial S_{g, l}\right]\right)$ is very direct and all powers of $y_{2}$ are untouched in this process. It follows that $\phi_{*}\left(\left[\partial S_{g, l}\right]\right)$ and $\phi_{*}\left(\left[\partial S_{g, l^{\prime}}\right]\right)$ do not have the same cyclic reduced form when $l \neq l^{\prime}$. Hence, if $l \neq l^{\prime}, S_{g, l}$ and $S_{g, l^{\prime}}$ are not homotopic in $H$, and therefore, they are not isotopic in $\partial H$.

Now, $S_{g, l}$ separates $H$ into two components $P_{1}$ and $P_{2}$, with $\partial P_{1}=$ $T_{1} \cup S_{g, l}$ and $\partial P_{2}=T_{2} \cup S_{g, l}$, where $T_{1} \cup T_{2}=\partial H$ and $\partial T_{1}=\partial T_{2}=\partial S_{g, l}$.

Lemma 3.4. $T_{i}$ is incompressible in $H$.

Proof. Let $\phi_{\#}: H_{1}\left(S_{g, l}, Z\right) \rightarrow H_{1}(H, Z)$ be the induced homomorphism on the first homology groups. Note that $H_{1}(H, Z)=Z+Z+Z$ generate $\bar{y}_{1}$, $\bar{y}_{2}$ and $\bar{y}_{3}$, where $\bar{y}_{i}=\pi\left(y_{i}\right)$, and $\pi: \pi_{1}(H, Z) \rightarrow H_{1}(H, Z)$ is the abelization. By (3.0)-(3.4), it is easy to see that $i_{\#}\left(H_{1}\left(S_{g, l}, Z\right)\right)$ is a subgroup of $H_{1}(H, Z)$ generated by $4 \bar{y}_{1},(l+1) \bar{y}_{2}, \bar{y}_{3}$. Thus, $H_{1}(H, Z) / \phi_{\#}\left(H_{1}\left(S_{g, l}, Z\right)\right)$ is a finite group (of order $4 l+4$ ). 
If $T_{i}, i=1$ or 2 , is compressible, then there is a compressing disk $B_{1}^{\prime}$ in $H$ for $T_{i}$. Since $\partial B_{1}^{\prime} \cap \partial S_{g, l}=\emptyset$ and $S_{g, l}$ is incompressible in $H$, by standard argument in 3-manifold topology, we may assume that $B_{1}^{\prime} \cap S_{g, l}=\emptyset$. Furthermore, since $H$ is a handlebody, we may also assume that $B_{1}^{\prime}$ is nonseparating in $H$. Thus, there are two properly embedded disks $B_{2}^{\prime}$ and $B_{3}^{\prime}$ in $H$ such that, $\left(B_{1}^{\prime}, B_{2}^{\prime}, B_{3}^{\prime}\right)$ is a set of basis disks of $H$. Let $z_{1}, z_{2}$ and $z_{3}$ be generators of $\pi_{1}(H)$ corresponding to $B_{1}^{\prime}, B_{2}^{\prime}$ and $B_{3}^{\prime}$. Since $S_{g, l}$ misses $B_{1}^{\prime}, \phi_{*}\left(\pi_{1}\left(S_{g, l}\right)\right) \subset G \subset \pi_{1}(H)$, where $G$ is generated by $z_{2}$ and $z_{3}$. Then, clearly, $H_{1}(H, Z) / \phi_{\#}\left(H_{1}\left(S_{g, l}, Z\right)\right)$ is infinite group, a contradiction.

Jaco's Lemma [4]. Let $M$ be a compact 3-manifold with compressible $\partial M$ and $r$ be a circle in $\partial M$. If $\partial M-r$ is incompressible in $M$, then either $M[r]$ is a 3-ball or $\partial M[r]$ is incompressible.

Proof of Proposition 3.2. Since $S_{g, l}$ is incompressible in $H$ by Lemma 3.3 , and $H$ contains no closed incompressible surface, $\partial P_{i}$ is compressible in $P_{i}, i=1,2$;

Since $T_{1}, T_{2}$ and $S_{g, l}$ are incompressible in $H$ by Lemma 3.4 and Lemma 3.3, $T_{i}$ and $S_{g, l}$ are incompressible in $P_{i}$. Hence $\partial P_{i}-\partial S_{g, l}$ is incompressible in $P_{i}, i=1,2$. Since, clearly, $P_{i}\left[\partial S_{g, l}\right]$ is not a 3 -ball, $\partial P_{i}\left[\partial S_{g, l}\right]$ is incompressible by Jaco's Lemma. It follows that $\hat{S}_{g, l}$, which is parallel to a component of $\partial P_{i}\left[\partial S_{g, l}\right]$, is incompressible in $P_{i}\left[\partial S_{g, l}\right]$. Since $H\left[\partial S_{g, l}\right]$ is a union of $P_{1}\left[\partial S_{g, l}\right]$ and $P_{2}\left[\partial S_{g, l}\right]$ along $\hat{S}_{g, l}, \hat{S}_{g, l}$ is incompressible in $H\left[\partial S_{g, l}\right]$. Therefore, $\hat{S}_{g, l}$ is incompressible in $H_{K}\left[\partial S_{g, l}\right]$. We proved Proposition 3.2 (1).

Proposition 3.2 (2) follows Lemma 3.3. (2)

\section{4. $H_{k}$ is irreducible, $\partial$-irreducible, anannular.}

Recall $E_{j}, F_{j}, J_{k}, M_{k}, B_{k}, a_{i}, A_{i}, D_{i}, T, \mu$ defined in Section 2.

Lemma 4.1. $F_{1} \cup F_{2}$ is incompressible and $\partial$-incompressible in $H_{K}$.

Proof. Suppose first $F_{1} \cup F_{2}$ is compressible in $H_{K}$. Then there is a disk $B \subset M_{k}$ such that $B \cap\left(F_{1} \cup F_{2}\right)=\partial B$ and $\partial B$ is a non-trivial circle on $F_{1} \cup F_{2}$. Denote by $B^{\prime}$ the disk bounded by $\partial B$ in $E_{1} \cup E_{2}$. Then, $B \cup B^{\prime}$ is a 2-sphere $S^{2}$ in the solid torus $J_{k}$, so $B \cup B^{\prime}$ bounds a 3 -ball $B^{3}$ in $J_{k}$. Since $\partial B$ is non-trivial in $F_{1} \cup F_{2}, B^{\prime}$ contains $\partial_{1} a_{i}$ for some $a_{i} \subset J_{k}$. Since $S^{2}$ is separating and $a_{i}$ is connected, we must have $\left(a_{i}, \partial a_{i}\right) \subset\left(B^{3}, B^{\prime}\right)$, which provides a relative homotopy on $\left(J_{k}, E_{1} \cup E_{2}\right)$ sending $a_{i}$ to $E_{1} \cup E_{2}$, see Figure 7 (a), which contradicts Lemma 2.3 (2). 


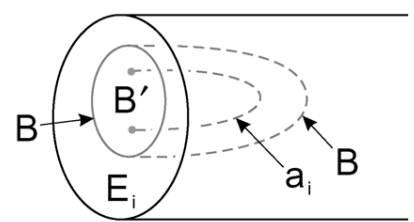

(a)

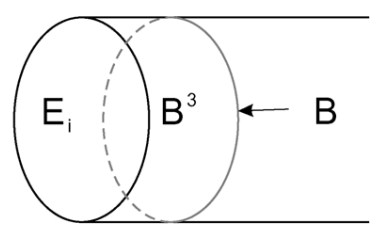

(c)

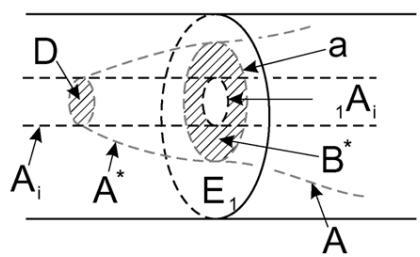

(e)

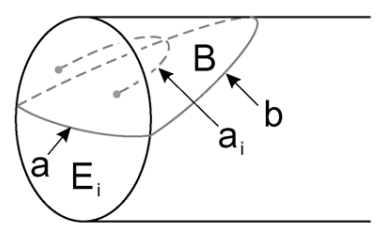

(b)

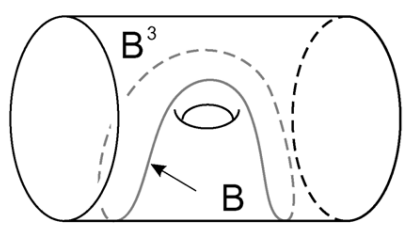

(d)

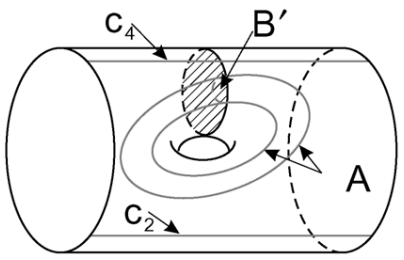

(f)

Figure 7.

Suppose then $F_{1} \cup F_{2}$ is $\partial$-compressible in $H_{K}$. Then there is a nontrivial arc $a$ in $F_{1} \cup F_{2}$ and an $\operatorname{arc} b$ in $\partial H_{K}$ bound a proper disk $B$ in $M_{k}$. There are two cases:

(1) $b \subset T$. Then $b$ is a proper arc in $A_{i}, i=1,5,3,7$. Now, either $b$ is a trivial arc in $A_{i}$, then there is an arc $b^{\prime}$ in $\partial A_{i}$ such that the circle $a \cup b^{\prime}$ is non-trivial in $F_{1} \cup F_{2}$ but bounds a disc in $M_{k}$, which contradicts the incompressibility of $F_{1} \cup F_{2}$ we just proved; or $b$ is a non-trivial arc in $A_{i}$, then the disc $B$ provides a relative homotopy on $\left(J_{k}, E_{1} \cup E_{2}\right)$ sending $a_{i}$ to $E_{1} \cup E_{2}$, which contradicts Lemma 2.3 (2).

(2) $b \subset \partial H$. Since $\left|B \cap\left(E_{1} \cup E_{2}\right)\right|=1, B$ separates a 3 -ball $B^{3}$ from $J_{k}$ by Lemma 2.3 (4). Since $a$ is non-trivial in $F_{1} \cup F_{2}$, by the same reason as the end of the first paragraph, one of $a_{i}$ lies in $B^{3}$ with $\partial a_{i}$ lies in a disc in $\partial B^{3} \cap E_{1}$, see Figure 7 (b), which contradicts Lemma 2.3 (2).

Lemma 4.2. $H_{K}$ is irreducible. 
Proof. Otherwise, there is an essential 2-sphere $S^{2}$ in $H_{K}$. Since $H$ is irreducible, $S^{2}$ bounds a 3 -ball $B^{3}$ in $H$ with $K \subset B^{3}$, which contradicts Lemma $2.3(1)$.

Lemma 4.3. $H_{K}$ is $\partial$-irreducible.

Proof. Suppose $H_{K}$ is $\partial$-reducible. Let $B$ be a compressing disk of $\partial H_{K}$. If $\partial B \subset T$, then $H_{K}$ contains an essential 2-sphere, which contradicts Lemma 4.2. Below, we assume that $\partial B \subset \partial H$. Furthermore, we assume that

$\left(^{*}\right)\left|B \cap\left(F_{1} \cup F_{2}\right)\right|$ is minimal among all compressing disks $B$ of $\partial H_{k}$.

Suppose first $B \subset M_{k}$. Since $B \cap\left(E_{1} \cup E_{2}\right)=\emptyset, B$ separates a 3 -ball $B^{3}$ from $J_{k}$ by Lemma $2.3(4)$. Since $\partial B$ is non-trivial in $\partial H_{K}$, then either $B^{3}$ contains only one of $E_{1}$ and $E_{2}$, see Figure 7 (c), and then $\left\|a_{i}, B\right\| \neq \emptyset$ for all $a_{i} \subset J_{k}$, a contradiction; or $B^{3}$ contains both $E_{1}$ and $E_{2}$ and $k=2$ in this case, see Figure $7(\mathrm{~d}), a_{4}$ and $a_{8}$ are properly homotopic in $\left(B^{3}, E_{1} \cup E_{2}\right) \subset$ $\left(J_{k}, E_{1} \cup E_{2}\right)$, which contradicts Lemma $2.3(2)$.

Suppose then $B \cap\left(F_{1} \cup F_{2}\right) \neq \emptyset$. By Lemma 4.1 and the assumption $\left(^{*}\right)$, $B \cap\left(F_{1} \cup F_{2}\right)$ consists of arcs. Then an outmost arc $a$ of $B \cap\left(F_{1} \cup F_{2}\right) \subset B$ separates a disk $B_{0}$ from $B$ with $B_{0} \subset M_{k}$ for some $k$. By (*) a must be non-trivial in $F_{1} \cup F_{2}$, (otherwise $\left|B \cap\left(F_{1} \cup F_{2}\right)\right|$ can be reduced by pushing $B_{0}$ to a suitable side). Since $\left|B_{0} \cap\left(E_{1} \cup E_{2}\right)\right|=1, B_{0}$ separates a 3 -ball from $J_{k}$ by Lemma $2.3(4)$. Then we reach a contradiction by the same reason in the end of the proof of Lemma 4.1

Lemma 4.4. $M$ is anannular.

Proof. Suppose $H_{K}$ contains an essential annulus $A$. Assume that

$(* *)\left|A \cap\left(F_{1} \cup F_{2}\right)\right|$ is minimal among all essential annuli in $H_{K}$.

By Lemma 4.1 and $\left.{ }^{* *}\right)$, each component of $A \cap\left(F_{1} \cup F_{2}\right)$ is non-trivial in both $A$ and $\left(F_{1} \cup F_{2}\right)$. There are three cases:

Case 1. $\partial A \subset T$. There are two sub-cases:

(1) $\left\|\partial_{1} A, \mu\right\|=0$. Let us assume $\partial A \cap\left(F_{1} \cup F_{2}\right)=\emptyset$.

Suppose first $A \cap\left(F_{1} \cup F_{2}\right)=\emptyset$. Let us assume that $A$ is contained in $M_{2}$ (the remaining cases are more direct). The whole $\partial A$ must lie in the same $A_{i} \subset M_{2}$ by Lemma 2.3 (3). Since $A$ is essential, $A \cap D_{i}=\emptyset$ for $i=2,4,8$. Then $\partial A \subset A_{6}$. Let $M^{\prime}$ be obtained by cutting $M_{2}$ along $D_{2}, D_{4}, D_{8}$. Then $A$ is still an incompressible annulus in $M^{\prime}$, and $D_{6} \subset M_{2}$ become a properly embedding disk $D_{6}^{\prime} \subset M^{\prime}$ with $\partial D_{6}^{\prime} \cap A_{6}=\partial D_{6} \cap A_{6}$, a non-trivial arc of $A_{6}$. Since $\partial A \subset A_{6}, A \cap D_{6}^{\prime}$ is an arc in both $A$ and $D_{6}^{\prime}$. Hence, there is a 
$\partial$-compressing disk of $A$ in $M^{\prime}$ which is also a $\partial$-compressing disk of $A$ in $M_{2}$, which contradicts that $A$ is essential in $M_{2}$.

Suppose then $A \cap\left(F_{1} \cup F_{2}\right) \neq \emptyset$, which must be a union of circles. An outmost circle $a$ of $A \cap\left(F_{1} \cup F_{2}\right) \subset A$ and $\partial_{1} A$ bound an annulus $A^{*} \subset A$. May assume that $a \subset F_{1}$, and $\partial_{1} A \subset A_{i} \subset M_{k}$. Let $B^{*}$ be the disk bounded by $a$ on $E_{1}$ and $D$ be the meridian disk of $N(K)$ bounded by $\partial_{1} A$. $B^{*} \cup A^{*} \cup D$ is a separating 2-sphere $S^{2}$ which bounds a 3-ball $B^{3} \subset J_{k}$, see Figure 7 (e). Since $\left.\left|\left(A^{*} \cup D\right) \cap\left(\cup_{a_{j} \subset J_{k}} a_{j}\right)\right|=\mid\left(A^{*} \cup D\right) \cap a_{i}\right) \mid=1$, by applying Lemma 2.3 (2) as before, we have $\left|B^{*} \cap\left(\cup_{a_{j} \subset J_{k}} a_{j}\right)\right|=\left|B^{*} \cap a_{i}\right|=1$. Hence, $a$ and $\partial_{1} A_{i}$ bound an annulus $A^{\prime}$ in $F_{1}$. Now, by pushing the annulus $\overline{A-A^{*}} \cup A^{\prime}$ to a suitable side of $F_{1},\left|A \cap\left(F_{1} \cup F_{2}\right)\right|$ is reduced, which contradicts (**).

(2) $\left\|\partial_{1} A, \mu\right\| \geq 1$. Now, $A \cap\left(F_{1} \cup F_{2}\right)$ consists of non-trivial arcs in $A$, which cut $A$ into $8\left\|\partial_{1} A, \mu\right\|$ rectangles and each rectangle has two opposite edges on $F_{1} \cup F_{2}$ and two opposite edges on $A_{i}$ and $A_{\pi(i)}$, where $\pi(i)=$ $i+l \bmod 8$. If $l=0$, then the two ends of each arc of $A \cap\left(F_{1} \cup F_{2}\right)$ lie in a same component of $\partial\left(F_{1} \cup F_{2}\right)$, and an inner most arc is trivial in $F_{1} \cup F_{2}$, a contradiction [2]. If $l \neq 0 \bmod 8$, then $a_{6}$ and $a_{6+l}$ are properly isotopy in $M_{2}$, which contradicts to Lemma $2.3(2)$.

Case 2. $\partial_{1} A \subset T$ and $\partial_{2} A \subset \partial H$.

By Lemma 4.3, both $\partial H$ and $T$ are incompressible in $H_{K}$. Clearly, $H_{K}$ is not homeomorphic to $T \times I$. Since both Dehn fillings along $\mu$ and $\partial A_{1}$ compress $\partial H_{K}$, by $[1,2.4 .3], \Delta\left(\partial_{1} A, \mu\right) \leq 1$. There are two sub-cases.

(1) $\left\|\partial_{1} A, \mu\right\|=0$. Since $\partial_{1} A$ is disjoint from $F_{1} \cup F_{2}, \partial_{2} A$ is disjoint from $F_{1} \cup F_{2}$ (otherwise, there is an arc in $A \cap\left(F_{1} \cup F_{2}\right)$ with two ends in $\partial_{2} A$ which is trivial in $A$ ). Then it follows that $A$ is disjoint from $F_{1} \cup F_{2}$ by the proof of Case 1. Suppose $\partial_{1} A \subset A_{i} \subset M_{k}$ for some $i, k$. Let $D$ be the meridian disk of $N(K)$ bounded by $\partial_{1} A$ and $B=A \cup_{\partial_{1} A} D$. Then $B$ is a proper disc in $J_{k}, \partial B$ is non-trivial in $\partial H \cap J_{k}$, and $\left|B, \cup_{a_{j} \subset M_{k}} a_{j}\right|=\left|B, a_{i}\right|=1$. Since $B \cap\left(E_{1} \cup E_{2}\right)=\emptyset, B$ separates a 3 -ball $B^{3}$ from $J_{2}$ by Lemma 2.3 (4). If $B^{3}$ contains only one of $E_{1}$ and $E_{2}$, then $B$ meets all $a_{j}$ in $J_{k}$. If $B^{3}$ contains both $E_{1}$ and $E_{2}$, then $B$ meets $a_{i}$ in non-zero even number. In each case, we reach a contradiction.

(2) $\left\|\partial_{1} A, \mu\right\|=1$. It is easy to see that this case is ruled out by Lemma $2.3(5)$.

Case 3. $\partial A \subset \partial H$.

Suppose first $A \cap\left(F_{1} \cup F_{2}\right)=\emptyset$. Since $A$ is essential, $A$ is disjoint from $D_{i}$ for $i \neq 6$. Let us assume that $A \subset M_{2}$ (the remaining cases are the same). Since $\partial A \subset \partial H \cap J_{2}$ and $A$ is disjoint from $c_{4}, c_{2}, A$ separates $J_{2}$ into two solid torus $J^{*}$ and $J^{\prime}$ such that $\left(E_{1} \cup E_{2}\right) \cap J^{\prime}=\emptyset$ and separates a disc 
$B^{\prime} \subset J^{\prime}$ from $B_{2}$, see Figure 7 (f). Since $J^{\prime}$ is disjoint from all $A_{i} \subset M_{2}, A$ is $\partial$-compressible in $M_{2}$, which contradicts that $A$ is essential in $M_{2}$.

Suppose then $A \cap\left(F_{1} \cup F_{2}\right) \neq \emptyset$. There are two sub-cases:

(1) $A \cap\left(F_{1} \cup F_{2}\right)$ consists of circles. Then an outmost circle $a$ of $A \cap$ $\left(F_{1} \cup F_{2}\right) \subset A$ and $\partial_{1} A$ bound an annulus $A^{*} \subset A$ such that $A^{*} \subset M_{k}$. Let $B^{*}$ be the disk bounded by $a$ on $E_{1} \cup E_{2}$ and $D^{*}=A^{*} \cup B^{*}$. By slightly pushing, we have $D^{*} \subset J_{k}$, moreover, (i) $D^{*} \cap a_{i} \neq \emptyset$ for some $a_{i} \subset J_{k}$, (ii) for each $a_{j} \subset J_{k},\left|D^{*} \cap a_{j}\right| \leq 2$, and $\leq 1$ if $k=2$. Since $\partial D^{*} \subset J_{k} \cap \partial H, D^{*}$ separates a 3 -ball $B^{3}$ from $J_{k}$ by Lemma 2.3 (4). Now either $B^{3}$ contains both $E_{1}$ and $E_{2}, k=2$ in this case, and $D^{*}$ meets each $a_{j} \subset J_{2}$ in even number of points, which contradicts (i) and (ii) above; or $B^{3}$ contains only one $E_{i}$, say $E_{1}$, then $\partial_{1} A=\partial D^{*}$ is parallel to $\partial E_{1}$. Since $A^{*}$ is disjoint from $K,\left|B^{*} \cap\left(\cup_{a_{j} \subset J_{k}} a_{j}\right)\right|=\left|D^{*} \cap\left(\cup_{a_{j} \subset J_{k}} a_{j}\right)\right|=\left|E_{1} \cap\left(\cup_{a_{j} \subset J_{k}} a_{j}\right)\right|=4$. Hence, $a$ and $\partial E_{1}$ bound an annulus $A^{\prime}$ in $F_{1}$ by applying Jordan Curve Theorem. Now, we reach a contradiction by the same argument at the end of Case 1 (1).

(2) $A \cap\left(F_{1} \cup F_{2}\right)$ consists of arcs. Then $F_{1} \cup F_{2}$ cut $A$ into rectangles $R_{i}$, and each $R_{i}$ has two opposite sides in $F_{1} \cup F_{2}$ and remaining two sides in $\partial H$. Then $R_{i}$ separates a 3 -ball $B_{i}^{3}$ from $J_{k}$ by Lemma $2.3(4)$. Let $D_{i}^{1}$ and $D_{i}^{2}$ be two disks of $B_{i}^{3} \cap\left(E_{1} \cup E_{2}\right)$. By Lemma 2.3 (2), we have (i) $\partial_{1} a_{j} \subset D_{i}^{1}$ if and only if $\partial_{2} a_{j} \subset D_{i}^{2}$. (ii) $a_{j}$ and $a_{l}$ are contained in the same $B_{i}^{3}$ implies that $(j, l)=(2,8)$.

If $a_{2}$ and $a_{8}$ belong to the same $B_{i}^{3}$, then so do $a_{3}$ and $a_{7}$, which contradicts (ii). Hence, there is only one $a_{j}$ in each $B_{i}^{3}$. Hence, $A$ separates from $H$ a solid torus with $K$ as centerline (up to isotopy). Then $K$ and a component of $\partial A \subset \partial H$ bound an annulus, which contradicts Lemma 2.3 (5).

\section{5. $H_{K}$ contains no closed essential surfaces.}

Recall $W, W_{i}, l_{i}$ defined in Section 2 .

Suppose $H_{K}$ contains closed essential surfaces $F$. We define the complexity of $F$ by an ordered pair

$$
C(F)=\left(|F \cap W|,\left|F \cap\left(F_{1} \cup F_{2}\right)\right|\right) .
$$

Suppose $F$ realizes the minimality of $C(F)$. By the minimality of $C(F)$, Lemma 4.1 and the standard argument in 3-manifold topology, we have

Lemma 5.1. (1) Each component of $F \cap\left(F_{1} \cup F_{2}\right)$ is a non-trivial circle in both $F$ and $F_{1} \cup F_{2}$, 


\begin{tabular}{|c|c|c|c|c|c|c|}
\hline & & & & & \\
\hline & & & & & & \\
\hline & & & & & & \\
\hline & & & & & & \\
\hline & $W_{8}^{\prime}$ & $W_{i}$ & & & & $W_{5}$. \\
\hline$I_{7}$ & $\mathrm{I}_{8} \cdot$ & $I_{1} \cdot$ & $\mathrm{I}_{2}$ & $I_{3}$ & $\mathrm{I}_{4} \cdot$ & $\mathrm{I}_{5} \cdot$ \\
\hline & & & & & & 1 \\
\hline & & & & & & \\
\hline
\end{tabular}

Figure 8.

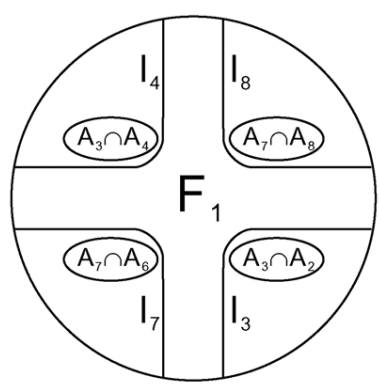

(a)

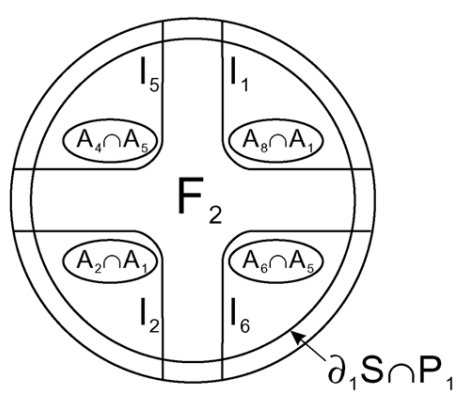

(b)

Figure 9.

(2) $F \cap W \subset W$ is a union of arcs as in Figure 8. Hence, $\left|F \cap l_{i}\right|=\left|F \cap l_{j}\right|$ for all $i, j$.

The positions of $\partial A_{i}$ and $l_{i}$ in $F_{1} \cup F_{2}$ are indicated as in Figure 9 .

Below, we will use $\tilde{s}$ to denote a given family of parallel disjoint proper 1manifolds on some surface, and use $s$ to denote a representive (a component) of $\tilde{s}$.

Lemma 5.2. Each component of $F \cap M_{k}$ is isotopic to either $M_{k} \cap \partial H$ or some $A_{i} \subset M_{k}$, where $k=1,3$.

Proof. The proofs for $k=1$ and 3 are the same. Assume $k=3$. First, we need 


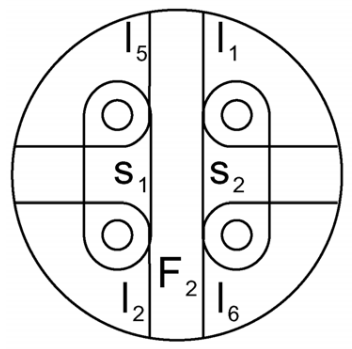

(a)

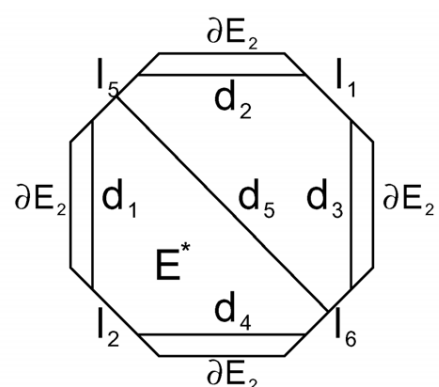

(b)

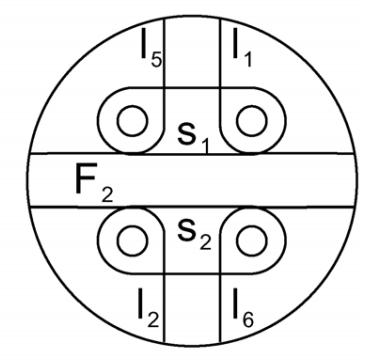

(c)

Figure 10.

Lemma 5.3. Components of $F \cap F_{2}$ in $F_{2}$ which are not parallel to a component of $\partial F_{2}$ are divided into two families of circles $\tilde{s}_{1}$ and $\tilde{s}_{2}$ in Figure 10 (a) and $(c)$. Moreover, in each case, $\left|\tilde{s}_{1}\right|=\left|\tilde{s}_{2}\right|$.

Proof. Since each component of $F \cap F_{2}$ isotopic to a component of $\partial F_{2}$ contributes the same to $\left|F \cap l_{k}\right|$ for all $l_{k} \subset F_{2}$, we may assume that $F \cap F_{2}$ contains no such components when we apply Lemma 5.1 (2) to prove Lemma 5.3.

Note that $l_{1}, l_{2}, l_{5}, l_{6}$ separate $F_{2}$ into four annuli and one disc which is presented as an octagon $E^{*}$ in Figure 10 (b), where $F \cap E^{*}$ are presented as five families of proper disjoint $\operatorname{arcs} \tilde{d}_{1}, \ldots, \tilde{d}_{4}, \tilde{d}_{5}$ with $\partial d_{i}$ in different $l_{j}$ and $l_{k}$ for each $i$. By Lemma 5.1 (2), we have

$$
\left|\tilde{d}_{4}\right|+\left|\tilde{d}_{1}\right|=\left|\tilde{d}_{2}\right|+\left|\tilde{d}_{1}\right|+\left|\tilde{d}_{5}\right|=\left|\tilde{d}_{2}\right|+\left|\tilde{d}_{3}\right|=\left|\tilde{d}_{4}\right|+\left|\tilde{d}_{3}\right|+\left|\tilde{d}_{5}\right| .
$$

It follows $\left|\tilde{d}_{5}\right|=0,\left|\tilde{d}_{1}\right|=\left|\tilde{d}_{3}\right|$ and $\left|\tilde{d}_{2}\right|=\left|\tilde{d}_{4}\right|$. Back to Figure 9 (b), since no component is isotopic to $\partial F_{2}$, it follows that either $\left|\tilde{d}_{2}\right|=0$, which is Figure $10(\mathrm{a})$, or $\left|\tilde{d}_{1}\right|=0$, which is Figure 10 (c).

Let us return to the proof of Lemma 5.2. Let $S$ be a component of $F \cap M_{3}$. Each $W_{i}$ separates a solid tori $P_{i}$ from $M_{3}, i=1,5$. Let $M_{3}^{\prime}=$ $\overline{M_{3}-\left(P_{1} \cup P_{5}\right)}$, which is a solid torus. There are three cases to discuss.

Case 1. If a component of $\partial S$ is isotopic to a component of $\partial A_{i}, i=$ 1,5. By the minimality of the complexity $C(F), S$ is disjoint from $W_{i}$, and therefore $S \subset P_{i}$, which is an annulus isotopy to $A_{i}, i=1$ or 5 .

Case 2. If a component of $\partial S$ is isotopic to $\partial E_{2}$, let $\partial_{1} S$ be the outmost component of $\partial S \subset F_{2}$ which is isotopic to $\partial E_{2}$. Now, $\partial_{1} S$ intersects $P_{i}$ 


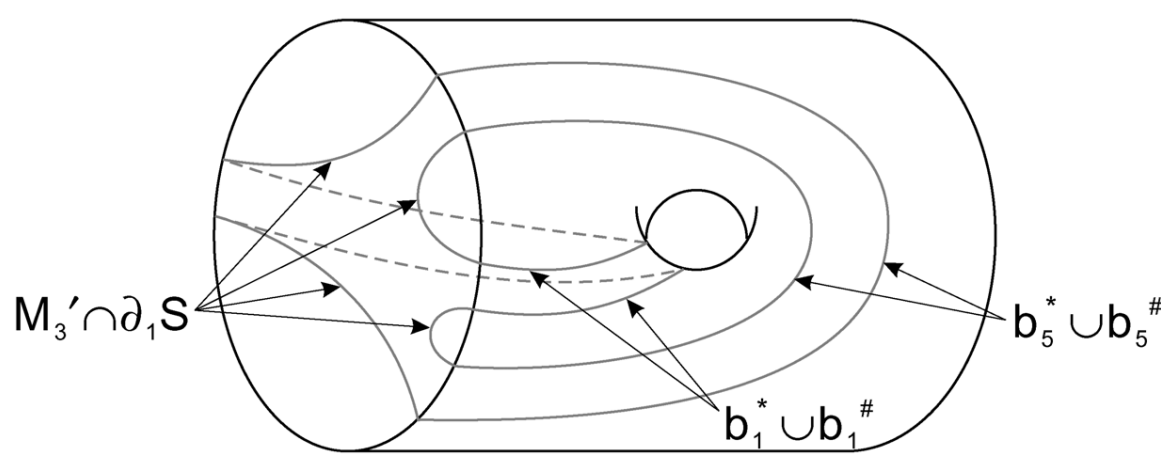

Figure 11.

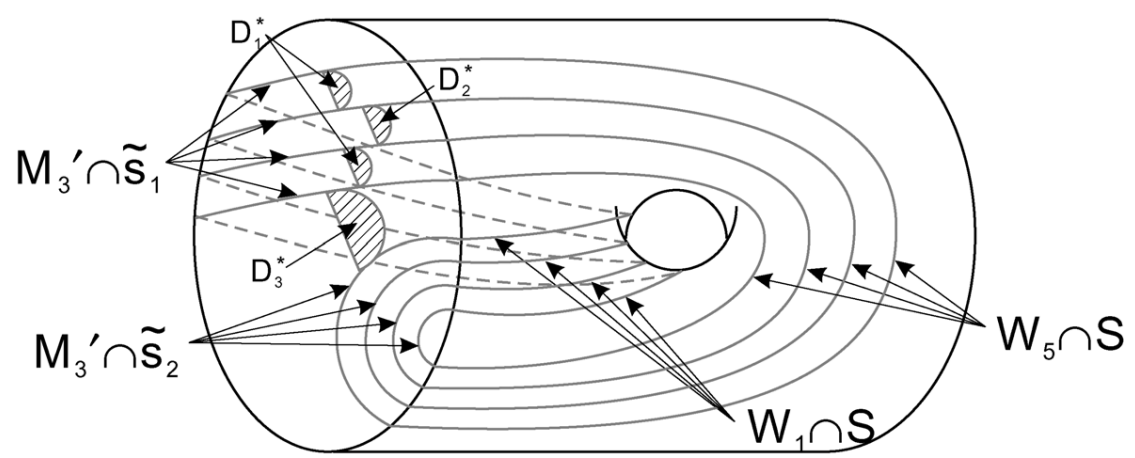

Figure 12 .

as in Figure 9 (b) and $W_{i} \cap S$ contains two $\operatorname{arcs} b_{i}^{*}$ and $b_{i}^{\#}$ with ends in $\partial_{1} S$. Let $S_{i}$ be the component of $S \cap P_{i}$ which meets $\partial_{1} S, i=1,5$. Then $S_{i}$ is incompressible in $P_{i}$. Since $\partial S_{i}=\left(\partial_{1} S \cap P_{i}\right) \cup\left(b_{i}^{*} \cup b_{i}^{\#}\right)$ bounds a disk in $P_{i}$ parallel to $\partial M_{3}, S_{i}$ itself is such a disc, $i=1,5$. Let $S_{3}$ be a component of $S \cap M_{3}^{\prime}$ which meets $\partial_{1} S$, then $S_{3}$ is incompressible in the solid torus $M_{3}^{\prime}$ and $\partial S_{3}$ has a component $\left(\partial_{1} S \cap M_{3}^{\prime}\right) \cup\left(b_{1}^{*} \cup b_{1}^{\#}\right) \cup\left(b_{5}^{*} \cup b_{5}^{\#}\right)$ which bounds a disk in $\partial M_{3}^{\prime}$ as in Figure 11. Hence, $S_{3}$ itself is such a disk. Thus $S=S_{1} \cup_{b_{1}^{*} \cup b_{1}^{\#}} S_{3} \cup_{b_{5}^{*} \cup b_{5}^{\#}} S_{5}$ is isotopic to $M_{3} \cap \partial H$.

By Lemma 5.3, to finish the proof, we need only to rule out Case 3 below.

Case 3. $\left|\tilde{s}_{1}\right|=\left|\tilde{s}_{2}\right| \neq 0$ in Figure 10 (a) or (b). Since the discussion for (a) and (c) in Figure 10 are the same, we just discuss the former case.

We may assume that no component of $S$ is isotopic to a component of $\partial F_{2}$ by Case 1 and Case 2 we just discussed. Let $S_{3}^{\prime}=S \cap M_{3}^{\prime}$. Then $\partial S_{3}^{\prime}$ 


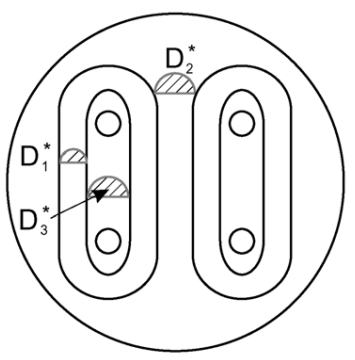

(a)

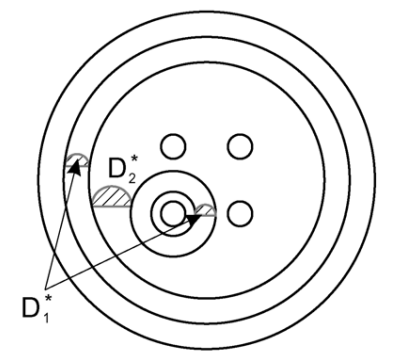

(b)

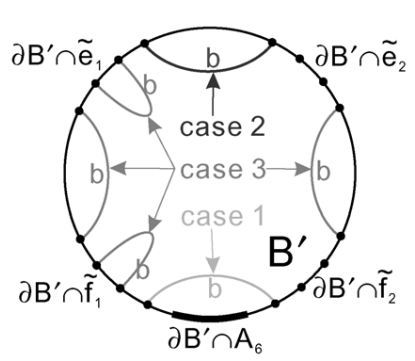

(c)

Figure 13.

contains $2\left|\tilde{s}_{1}\right|$ circles which are produced from the arcs $\left(\tilde{s}_{1} \cup \tilde{s}_{2}\right) \cap M_{3}^{\prime}$ and the arcs $\left(W_{1} \cup W_{5}\right) \cap S$, as in Figure 12, where $\left|\tilde{s}_{1}\right|=2$. Note each circle in $\partial S_{3}^{\prime}$ is non-trivial in $\partial M_{3}^{\prime}$. Since $S_{3}^{\prime}$ is incompressible in the solid torus $M_{3}^{\prime}$, each component of $S_{3}^{\prime}$ is an annulus which is $\partial$-compressible. Now, $B_{3}^{\prime} \cap S_{3}^{\prime}$ is a union of arcs, where $B_{3}^{\prime}=B_{3} \cap M_{3}^{\prime}$. An outmost arc $b$ of $B_{3}^{\prime} \cap S_{3}^{\prime} \subset B_{3}^{\prime}$ separates a disc $D^{*}$ from $B_{3}^{\prime}$. As a $\partial$-compressing disc of $S_{3}^{\prime}, D^{*}$ can be moved into the positions of $D_{1}^{*}, D_{2}^{*}, D_{3}^{*}$, indicated as in Figure 12. Now, back to $M_{3}$, those $D_{i}^{*}$ s in Figure 12 are corresponding to those $D_{i}^{*}$ 's in Figure 13 (a), $i=1,2,3$. In the cases of $D_{1}^{*}$ and $D_{3}^{*}$ in Figure 13 (a), one can push $F$ along the disc to reduce $|F \cap W|$; in the case of $D_{2}^{*}$ in Figure 13 (a), one can push $F$ along the disc to reduce $\left|F \cap\left(F_{1} \cup F_{2}\right)\right|$, but not to increase $|F \cap W|$. In each case, it contradicts the minimality of $C(F)$.

Remark on Figures 11, 12 and 14. In Figure 11, to simplify the picture, $W_{1}$ does not meet $B_{3}$ in three arcs as it should be. But one verifies easily that this simplification does not affect the proof. The same remark is needed for Figures 12 and 14. Moreover in Figure 14, we only draw a representative $e_{i}$ for a families $\tilde{e}_{i}$ and so on.

Proposition 5.4. $H_{K}$ contains no closed essential surface.

Proof. Now, we consider $F \cap M_{2}$. Each component of $F \cap\left(F_{1} \cup F_{2}\right)$ is isotopic to a component of $\partial F_{2} \cup \partial F_{1}$ by Lemma 5.2. Applying Lemma 5.1 (2) again, we have $\left|\widetilde{\partial E_{2}}\right|=\left|\widetilde{\partial E_{1}}\right|$, where $\widetilde{\partial E_{i}} \subset F_{i}$ are components of $F \cap F_{i}$ isotopic to $\partial E_{i}, i=1,2$. Each $W_{i}$ separates a solid tori $P_{i}$ from $M_{2}, i=2,4,8$. Let $M_{2}^{\prime}=\overline{M_{2}-\left(P_{2} \cup P_{4} \cup P_{8}\right)}$, which is a handlebody of genus 2 . 


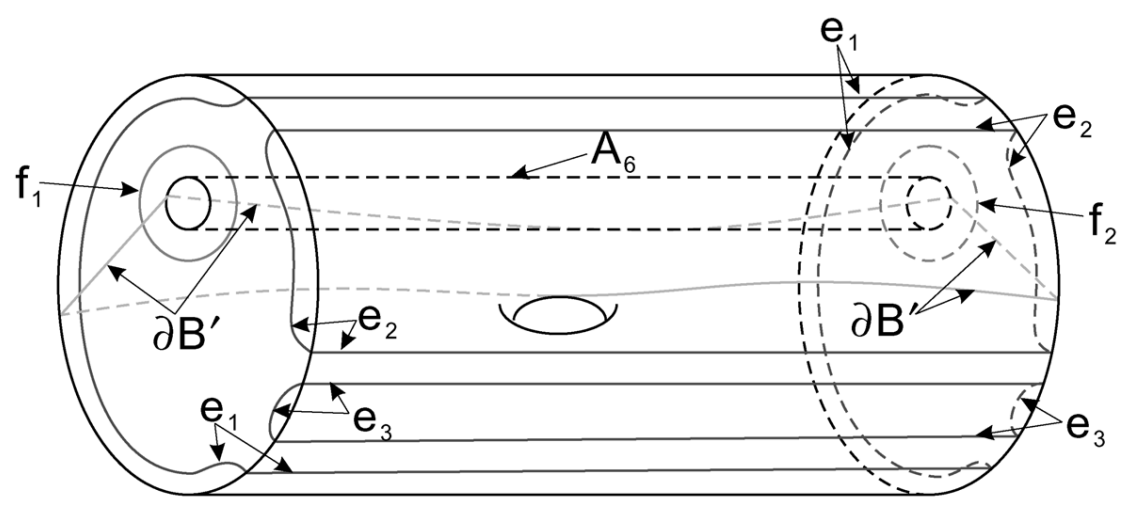

Figure 14 .

Note if $F \cap M_{2}$ has a component $S$ such that a component of $\partial S$ is isotopic to $\partial A_{i}, i=2,4,8$, by the minimality of $C(F), S \subset P_{i}$ and hence $S$ is isotopic to $A_{i}, i=2,4,8$.

Let $S_{2}^{\prime}=F \cap M_{2}^{\prime}$. Then $\partial S_{2}^{\prime}$ consists of possibly five families of circles $\tilde{e}_{1}, \tilde{e}_{2}, \tilde{e}_{3}, \tilde{f}_{1}$ and $\tilde{f}_{2}$, where $\tilde{e}_{1}, \tilde{e}_{2}$ and $\tilde{e}_{3}$ with $\left|\tilde{e}_{i}\right|=\left|\widetilde{\partial E_{1}}\right|$ are produced from the $\operatorname{arcs}\left(\widetilde{\partial E_{1}} \cup \widetilde{\partial E_{2}}\right) \cap M_{2}^{\prime}$ and the arcs of $\left(W_{2} \cup W_{4} \cup W_{8}\right) \cap F$ with end points lying $\widetilde{\partial E_{1}} \cup \widetilde{\partial E_{2}}, \tilde{f}_{1} \subset F_{1}$ and $\tilde{f}_{2} \subset F_{2}$ are parallel copies of the two components of $\partial A_{6}$ respectively. All those are indicated in Figure 14 (see Remark on Figures 11., 12, and 14). Moreover,

(i) each component of $\tilde{e}_{3}$ bounds a disk in $\partial M_{2}^{\prime}$, hence bounds also a disk in $F$.

(ii) any two components in $\tilde{e}_{1} \cup \tilde{e}_{2}$ bound an annulus in $\partial M_{2}^{\prime}$ disjoint from $A_{6}$.

There is a proper disc $B^{\prime}$ in $M_{2}^{\prime}$ with $\partial B^{\prime}$ shown in Figure 14 such that

(iii) $\partial B^{\prime}$ meets those four families in the cyclic order $\tilde{e}_{1}, \tilde{e}_{2}, \tilde{f}_{2}, \tilde{f}_{1}$,

(iv) $\partial B^{\prime}$ meets each component of $\tilde{e}_{1} \cup \tilde{e}_{2} \cup \tilde{f}_{1} \cup \tilde{f}_{2}$ in one point and $\partial B^{\prime} \cap A_{6}$ is a non-trivial arc in $A_{6}$,

Let $S^{\prime}$ be a component of $S_{2}^{\prime}$. Since $S^{\prime}$ is incompressible in $M_{2}^{\prime}, S^{\prime} \cap B^{\prime}$ consists of arcs. By (iv), there is an outmost arc $b$ of $S^{\prime} \cap B^{\prime} \subset B^{\prime}$ which separates a disk $D^{*}$ from $B^{\prime}$ so that

(v) $\partial D^{*}$ disjoint from $A_{6}$ and $D^{*}$ is a $\partial$-compressing disk of $S^{\prime}$.

We divide the remaining discussion into three cases by (iii). (Figure 13 (c) is helpful to understand (iii), (iv) and (v) above and each case below.)

Case 1. One end of $b$ is in $f_{1} \in \tilde{f}_{1}$ and the other is in $f_{2} \in \tilde{f}_{2}$. In this 
case, $\left|\widetilde{\partial E_{1}}\right|=0$ and all $\tilde{e}_{i}$ 's do not exist by (iii) and (v). One can show that $S^{\prime}$ is isotopic to $A_{6}$ by cutting and pasting argument in 3-manifold topology, the detail is contained in what we will do in Case 2.

Case 2. One end of $b$ is in $e_{1} \in \tilde{e}_{1}$ and the other is in $e_{2} \in \tilde{e}_{2}$. By (ii), $e_{1}$ and $e_{2}$ bound an annulus $A$ in $\partial M_{2}^{\prime}$ disjoint from $A_{6}$. Let $M_{2}^{\prime \prime}, S^{\prime \prime}, A^{\prime}$, $A_{6}^{\prime}, e_{1}^{\prime}, e_{2}^{\prime}$ be the images of $M_{2}^{\prime}, S^{\prime}, A, A_{6}, e_{1}, e_{2}$ respectively after cutting $M_{2}^{\prime}$ along $B^{\prime}$. By (iv), $e_{i}^{\prime}$ is an arc, $i=1,2$. Let $b_{i}, D_{i}^{*}, i=1,2$, be the two copies of $b$ and $D^{*}$ after cutting $M_{2}^{\prime}$ along $B^{\prime}$. By (v), the circle $c^{\prime} \subset \partial S^{\prime \prime}$ formed by four arcs $e_{1}^{\prime}, e_{2}^{\prime}, b_{1}^{\prime}$ and $b_{2}^{\prime}$ bound a disc $D_{1}^{*} \cup A^{\prime} \cup D_{2}^{*}$ in $\partial M_{2}^{\prime \prime}$ which is disjoint from $A_{6}^{\prime}$. Since $S^{\prime \prime}$ is incompressible in $M_{2}^{\prime \prime}, S^{\prime \prime}$ is such a disc up to isotopy. Back to $M_{2}^{\prime}, S^{\prime}$ is isotopic to the annulus $A \subset M_{2}^{\prime}$. Back to $M_{2}$, by (i) and similar argument in Case 2 in the proof of Lemma $5.2, S$ is isotopic to $M_{2} \cap \partial H$.

Case 3. If either $\partial b$ lie in one of the four families $\tilde{e}_{1}, \tilde{e}_{2}, \tilde{f}_{1}$ and $\tilde{f}_{2}$, or one end of $b$ is in $\tilde{e}_{i}$ and the other in $\tilde{f}_{i}, i=1$ or 2 , then $D^{*}$ can be moved in $M_{2}^{\prime}$ keeping to be a $\partial$-compressing disk of $S^{\prime}$ so that when we go back to $M_{2}$, it is a $\partial$-compressing disk of $F \cap M_{2}$ in the position of either $D_{1}^{*}$ or $D_{2}^{*}$ in Figure 13 (b). One can push $F$ along either $D_{1}^{*}$ or $D_{2}^{*}$ to reduce $C(F)$, which contradicts the minimality of $C(F)$. (Refer the end of the argument in Case 3 of the Proof of Lemma 5.2).

So, each component $S$ of $F \cap M_{2}$ is isotopic to either $M_{2} \cap \partial H$ or $A_{i}$, $i=2,4,6,8$. In the former case, $\partial S$ is $\partial E_{1}$ and $\partial E_{2}$ which bound (up to isotopy) $\partial H \cap M_{1}$ and $\partial H \cap M_{3}$ respectively by Lemma 5.2, and then $F$ is isotopic to $\partial H$. In the later case, by Lemma 5.2, each component of $F \cap\left(M_{1} \cup M_{3}\right)$ is an annulus isotopic to one of $A_{1}, A_{3}, A_{5}, A_{7}$. Since $F$ is closed, it follows that $F$ is a torus isotopic to $T$.

Proposition 3.1 follows from Lemmas 4.2, 4.3, 4.4 and Proposition 5.4. Hence, Theorem 1.2 is proved.

\section{Acknowledgement.}

We would like to thank Doctor Zheng Hao for all the figures in this paper. We would like to express our thanks to the referee for his comments on this paper. 


\section{References.}

[1] M. Culler, C. Gordon, J. Luecke and P. Shalen, Cyclic surgery on knots, Ann. of Math., 125(1987), 237-300.

[2] C. Gordon, Dehn filling: a survey. Knot theory (Warsaw, 1995), 129-144, Banach Center Publ., 42, Polish Acad. Sci., Warsaw, 1998.

[3] A. Hatcher, On the boundary curves of incompressible surfaces. Pacific J. Math. 99 (1982), 373-377.

[4] W. Jaco, Adding a 2-handle to a 3-manifold, An application to Property R, Proc. AMS 92 (1984), 288-292.

[5] M. Lackenby, Attaching handlebody to 3-manifolds, Geometry and Topology, Vol. 6 (2002), 889-904 (2002)

[6] R.F. Qiu, Incompressible surfaces in handlebodies and closed 3-manifolds of Heegaard genus two, Proc. AMS 128(2000), 3091-3097.

[7] M. Scharlemann and Y. Wu, Hyperbolic manifolds and degenerating handle additions. J. Aust. Math. Soc. (Series A) 55 (1993), 72-89.

[8] W. Thurston, Three dimensional manifolds, Kleinian groups and hyperbolic geometry. Bull. AMS, Vol. 6, (1982) 357-388.

Department of Mathematics

DALIAN University of TeChNology

116022, CHINA

E-mail address: qiurf@dlut.edu.cn

Department of Mathematics

Peking University

100871, CHINA

E-mail address: wangsc@math.pku.edu.cn

Received February 12, 2004. 